\title{
Analytical procedure for the design of PFRP-RC hybrid beams including shear interaction effects
}

\author{
Catalin A. Neagoe *, Lluís Gil \\ Department of Strength of Materials and Structural Engineering, Universitat Politècnica de Catalunya - BarcelonaTech, \\ Jordi Girona 31, 08034 Barcelona, Spain \\ Laboratory for the Technological Innovation of Structures and Materials (LITEM), Colon 11, TR45, Terrassa, 08222 \\ Barcelona, Spain
}

\section{Abstract}

Hybrid beams made of pultruded fiber-reinforced polymer (PFRP) shapes connected to reinforced concrete (RC) slabs are regarded as novel cost-effective and structurally-efficient elements. The current study addresses the need for a robust analytical procedure for the design of such members considering the structural implications of shear interaction effects. The discussed analytical procedure is based on the Timoshenko beam theory and on the elastic interlayer slip model extended from steel-concrete and timber-concrete composite beams, and presents the necessary mathematical tools for evaluating deflections, flexural capacities and stress distributions of hybrid beams. Partial interaction effects are quantified by using a proposed dimensionless parameter that depends mainly on the connection's stiffness. The analytical equations were validated successfully against available experimental data and conclusions indicate that the simplified model for partial interaction is viable and should be used even for specimens with full interlayer shear capacity.

\section{Keywords}

Hybrid beam; pultruded FRP; flexural behavior; analytical model; partial interaction.

\begin{tabular}{|ll|}
\hline \multicolumn{2}{|l|}{ Nomenclature } \\
$A$ & sectional area \\
$A_{c}$ & area of the concrete slab \\
$A_{p}$ & area of the PFRP profile \\
$A_{f}$ & area of the profile's flange \\
$A_{w}$ & area of the profile's web \\
$b_{c}$ & width of the concrete slab \\
$b_{f}$ & width of the profile's flange \\
$b_{p}$ & width of the PFRP profile \\
$b$ & distance between support and applied load \\
\hline
\end{tabular}

* Corresponding author. Tel.: +34 937398727.

E-mail address: catalin.andrei.neagoe@upc.edu (C.A. Neagoe). 


\begin{tabular}{|c|c|}
\hline$d$ & diameter of connector \\
\hline$d_{c}$ & distance between centroids of layers \\
\hline$d_{w}$ & depth of profile web \\
\hline E & modulus of elasticity \\
\hline EI & flexural stiffness \\
\hline$F$ & force \\
\hline$f_{c}$ & concrete strength \\
\hline G & shear modulus \\
\hline$G A$ & shear stiffness \\
\hline$h$ & height of the hybrid beam \\
\hline$h_{c}$ & height of the concrete slab \\
\hline$h_{p}$ & height of the PFRP profile \\
\hline$I^{2}$ & principal moment of inertia \\
\hline$K_{c}$ & connector shear stiffness \\
\hline$L$ & beam span \\
\hline$M$ & internal bending moment acting on the whole section \\
\hline$M_{j}$ & internal bending moment acting on layer $j$ \\
\hline$N$ & internal normal force acting on layers \\
\hline$P_{\max }$ & connector capacity \\
\hline$Q$ & concentrated load \\
\hline$q$ & distributed load \\
\hline$q_{0}$ & uniformly distributed load \\
\hline$s$ & slip \\
\hline$S$ & first moment of area \\
\hline$S L S$ & Serviceability Limit State \\
\hline$s_{C}$ & spacing of connectors \\
\hline$t_{f}$ & thickness of flange \\
\hline$t_{w}$ & thickness of web \\
\hline$U L S$ & Ultimate Limit State \\
\hline$V$ & internal shear force acting on the whole section \\
\hline$V_{j}$ & internal shear force acting on layer $j$ \\
\hline$w$ & deflection in the $Z$ direction \\
\hline$x$ & longitudinal coordinate \\
\hline$x_{r}$ & relative longitudinal coordinate \\
\hline$x_{u}$ & depth of neutral axis at ULS \\
\hline$z$ & depth coordinate \\
\hline \multicolumn{2}{|c|}{ Greek letters } \\
\hline$\alpha$ & parameter \\
\hline$\alpha L$ & composite action parameter \\
\hline$\beta$ & parameter \\
\hline$\Delta$ & variation/percentile difference, as specified \\
\hline$\varepsilon_{c, u}$ & ultimate compressive strain of concrete \\
\hline$\varepsilon_{s}$ & slip strain \\
\hline$\eta$ & degree of partial interaction acc. to EC4 \\
\hline$\kappa$ & Timoshenko shear coefficient \\
\hline$\mu$ & buckling length coefficient \\
\hline$\xi$ & partial interaction parameter \\
\hline$\xi_{A, B, C, D}$ & approximate partial interaction parameters \\
\hline$\xi_{i}$ & exact partial interaction parameter for static case $i$ \\
\hline$\sigma$ & normal stress \\
\hline$\tau$ & shear stress \\
\hline$\phi$ & curvature of hybrid beam \\
\hline$\varphi$ & $E I_{c o} / E I_{0}-1$ \\
\hline
\end{tabular}




\begin{tabular}{|c|c|}
\hline \multicolumn{2}{|c|}{ Subscripts and superscripts } \\
\hline $1,2,3$ & reference to static case \\
\hline A,B,C,D & reference to approximate formulation \\
\hline an & analytical \\
\hline $\exp$ & experimental \\
\hline$c$ & concrete \\
\hline$p$ & profile \\
\hline$i$ & reference to static case 1,2 or 3 \\
\hline$j$ & reference to material layer: concrete $c$, or profile $p$ \\
\hline $\mathrm{CO}$ & complete interaction \\
\hline$p a$ & partial interaction \\
\hline eff & effective \\
\hline$b$ & due to bending \\
\hline $\operatorname{sh}$ & due to shear \\
\hline$t$ & total \\
\hline$u$ & ultimate, at ULS \\
\hline $\max$ & maximum \\
\hline
\end{tabular}

\section{Introduction}

While a large sector of the composites' construction market is still devoted to strengthening applications, in the past two decades new structures for road and pedestrian bridges, marine piers or buildings were created by including from the very beginning composites that play a key role in their performance. In the aforementioned new structures pultruded FRP shapes, or PFRPs, are commonly used because of their reduced cost of manufacturing, low maintenance requirements and resemblance to standard steel profiles, in addition to their inherent high strength-to-weight ratio and resistance to aggressive environmental factors.

Studies [1,2] have shown that the profiles alone, although strong, are more susceptible to failure due to instability and high flexibility. Therefore, researchers have started to combine PFRP shapes with traditional materials such as reinforced concrete in order to obtain hybrid members with superior structural characteristics [3-9]. The connection between the two materials can be realized with mechanical joints, bonded joints or combined joints, depending on the design considerations. Mechanical connections are usually preferred due to the ease of inspection and disassembly, the short time they take to fully develop their strength capacity and to the ductility characteristics they can possess. In contrast, bonded joints require special tools, materials and installation conditions, are difficult to inspect and disassemble, with temperature and humidity possibly affecting their strength. A bonded joint will have a higher connection stiffness but also a reduced post-elastic capacity compared to a mechanical connection.

One of the characteristics of hybrid/composite elements is that a certain degree of slip can develop at the interface, as exemplified in Fig. 1. The slip causes a reduction of the beam's flexural stiffness and thus an increase in bending flexibility.

Partial shear interaction relies on many factors such as the capacity and stiffness of the connection system, chemical bond at the interface, joint configuration and cracking of concrete [10,11]. For the design of composite steel-concrete members, the European Eurocode 4 [12] and the American AISC 360-10 [13] specifications take into account the degree of shear 
connection in calculating deflections and bending capacities of beams only based on the capacity of the installed connectors, however due to the flexibility of the joint partial interaction can still occur. Many researchers have studied this problem, especially for composite beams made of conventional materials like steel, concrete and timber. Girhammar et al. [14-16] analyzed the static and dynamic behavior of beam-column elements with interlayer slip and deducted exact and simplified first and second order formulations for the displacement functions and various internal actions for timberconcrete composite beams. Faella et al. [17,18] developed an “exact” displacement-based finite element model for steelconcrete composite beams with flexible shear connection and a simplified analytical procedure accounting for concrete slab cracking and the resulting tension stiffening effect, nonlinear connection behavior and the reduction of connection stiffness in hogging bending moment regions. Researching the shear slip effects in steel-concrete composite beams Nie et al. [19-21] proposed a simplified analytical model that was validated against experimental data and design code specifications. Frangi and Fontana [22] described an elasto-plastic model for timber-concrete composite beams with ductile connection that is based on the capacity of the connectors and not on their stiffness modulus. The model was compared to experimental data and further validated by Persaud and Symons [23]. Furthermore, Schnabl et al. [24] and $\mathrm{Xu}$ et al. [25,26] considered also the effect of the transverse shear deformation on displacements in each layer and concluded that shear deformations are more important to be evaluated for two-layer beams having a high connection stiffness, a high flexural-to-shear moduli ratio, and short span. Martinelli et al. [27] carried out a comparative study of analytical models for steel-concrete composite beams with partial interaction by employing a dimensionless formulation. Shear-rigid and shear-flexible models were considered using the Timoshenko beam theory and the study indicated possible threshold values beyond which certain effects become negligible.

In a straightforward manner, the same formulations can be extended and adapted for PFRP-RC beams with partial shear interaction effects. Nevertheless, studies performed on hybrid beams so far have seldom considered these effects, underestimating the real structural behavior. For instance, Sekijima et al. $[28,29]$ investigated the experimental flexural response of glass FRP-concrete beams mechanically connected with bolts and nuts arranged in a cross stitch pattern. Because the calculations were based on the Euler-Bernoulli beam theory, it was concluded after an additional finite element simulation that the shear deformation at the shear spans and the slip-off between the concrete slab and the upper flange of the pultruded FRP shape should have been considered in order to model better the real behavior. Biddah [30] studied the feasibility of using hybrid FRP-concrete beams instead of simple FRP profiles, by highlighting advantages such as increase in load carrying capacity and flexural stiffness. Separation between the connected layers was observed during the experimental tests, pointing out that the composite action was only partially developed. Thus, he concluded that the performance characteristics of the hybrid system need further investigation and development. The structural behavior of composite T-beams made of rectangular FRP tubes and concrete slabs was investigated by Fam and Skutezky [31]. The beam-slab specimens were connected with GFRP dowels forced into holes drilled on the top of the profiles. Stiffness degradation was observed, due to interface slippage, especially for hollow tube specimens. Correia et al. [32,33] took into consideration the shear slip effects for evaluating the analytical flexural response of GFRP-concrete hybrid beams, by using a simplified approach proposed by Knowles [34].

The current study presents an analytical procedure for the design of PFRP-RC hybrid beams including shear interaction effects. The flexural behavior of the hybrid elements is modeled using the Timoshenko beam theory and the connection is 
considered elastic with a uniform stiffness. In the procedure, the interaction effects are included only in the bending component of the Timoshenko composite beam model, after being evaluated for an equivalent shear-rigid composite beam. A dimensionless parameter is introduced to account for the degree of partial interaction, from the perspective of the connection's stiffness, and exact and approximate expressions are deducted for it. Exact and simplified formulations are also presented for calculating deflections, interlayer slip, bending capacities, and normal and shear stress distributions for hybrid beams under different interaction conditions. A validation of the analytical models is performed against published experimental data so as to assess the feasibility of using approximate solutions for partial interaction effects.

\section{Analytical models}

In the case of hybrid beams made of pultruded FRP profiles connected to reinforced concrete, the composite action in the structural members depends mainly on the shear behavior of the connection system. For achieving full shear interaction, high performance materials require more shear connectors, but because of the limited number that a top flange can accommodate for an optimal design and due to the stiffness of the connection, a partial composite design may be considered.

Consequently, the following section discusses analytical models suited for characterizing the short-term flexural behavior of hybrid beams under both complete and partial interaction situations. Mathematical expressions for evaluating deflections, slippage, flexural capacities and stress distributions are presented for a hybrid beam composed of an I-shaped pultruded FRP profile connected to a rectangular reinforced concrete slab, as illustrated in Fig. 2. The formulations can be extended to other prismatic, vertically symmetric cross-sections.

The composite profile is expected to behave elastically up to failure while the concrete has a typical nonlinear constitutive law as described in Eurocode 2 [35]. The orthotropic mechanical properties of the composite material are the same in the web and flanges, i.e. the profile's section is transversely isotropic. At the Ultimate Limit State (ULS), the concrete's compressive stress distribution is simplified as a rectangle characterized by parameters $\lambda$ and $n$, which are equal to 0.8 and 1.0 for concrete strength classes $\leq \mathrm{C} 50 / 60$. The depth of the neutral axis is designated $x_{u}$, the ultimate compressive strain $\varepsilon_{c, u}=3.5 \%$, the compressive strength of concrete $f_{c}$, and the slip strain developing at the interface is denoted $\varepsilon_{s}$. Material and design safety coefficients are not included herein and are to be found in specific design guides or manuals.

The scope of the analysis is restricted to beams subjected to positive bending so serviceability aspects (SLS) and failure criteria (ULS) are discussed for this specific case. In order to obtain closed-form solutions to the following analytical equations, three statically determinate beam cases are considered and depicted in Fig. 3. Other cases can be solved in a similar manner by applying the appropriate boundary conditions. The loads $Q, 2 Q$ and $q_{0}$ are applied over a span $L$ and the displacements (deflections) registered in the $Z$ direction are denoted with $w(\mathrm{x})$ for the corresponding coordinate along the $X$ axis. 


\subsection{Deflection}

\subsubsection{Complete interaction}

The analytical model of a PFRP-RC hybrid beam with complete shear interaction is based on the following assumptions:

- $\quad$ plane sections remain plane after deformation;

- there is no vertical separation or longitudinal slippage between the PFRP profile and the RC slab;

- the top steel reinforcement contribution is neglected;

- the whole width of the concrete slab is effective.

In addition, the evaluation of deflections is performed under the elastic range of the beam's constitutive materials because hybrid beams possess an inherent generally linear behavior until failure.

Due to the high ratio between the longitudinal elastic modulus and the shear modulus, for pultruded orthotropic composite materials it is necessary to consider also the shear deformation contributions in computing deflections by employing Timoshenko's beam theory. Thus, the elastic curve that describes the deflected shape of a hybrid PFRP-RC element is a function of its flexural rigidity $E I$ and transverse shear rigidity $\kappa G A$, so consequently the total deflection at a certain coordinate $w_{t}^{c o}(x)$ is expressed as a sum of the deflection due to bending deformation $w_{b}^{c o}(x)$ and the deflection due to shear deformation $w_{s h}(x)$ :

$$
w_{t}^{c o}(x)=w_{b}^{c o}(x)+w_{s h}(x)=\frac{f(x)}{E I_{c o}}+\frac{g(x)}{\kappa G A}
$$

where $f(x)$ and $g(x)$ are functions given by the elasticity theory which depend on the load and supporting conditions.

The flexural rigidity under complete interaction conditions can be obtained from the following relation:

$$
E I_{c o}=E I_{0}+\overline{E A} \cdot d_{c}^{2}
$$

where:

$$
\begin{aligned}
& E I_{0}=E_{c} I_{c}+E_{p} I_{p} \\
& \overline{E A}=\frac{E_{c} A_{c} \cdot E_{p} A_{p}}{E_{c} A_{c}+E_{p} A_{p}}
\end{aligned}
$$

where $E I_{0}$ represents the flexural rigidity when there is no shear interaction, $\overline{E A}$ the axial stiffness ratio and $d_{c}$ the distance between the centroids of areas $A_{c}$ and $A_{p}$. Variables $E_{c}$ and $E_{p}$ represent the longitudinal elastic moduli of the concrete and the profile (effective value) while $I_{c}$ and $I_{p}$ are the principal moments of inertia of the two. If the slab cracks under tension, only the compressed concrete area should be considered for calculating $I_{c}$.

When it comes to the shear rigidity of a hybrid beam, based on the fact that the majority of the shear stress in a composite member develops in the web of the profile, it can be assumed in a conservative approach that its effective value can be approximated as: 


$$
\kappa G A \approx G_{p} A_{w}
$$

where $\kappa$ is the Timoshenko shear coefficient, $G A$ transverse shear rigidity of the beam, $G_{p}$ the effective in-plane shear modulus of the FRP profile, and $A_{w}$ is the profile's web area. Several studies [36-40] have proposed exact or approximate expressions for computing the shear coefficient for orthotropic PFRP profiles but nevertheless they prove to be too complex for current use. In the end, the differences between the exact and approximate methods of determining the transverse shear rigidity for an I-shaped profile have been reported in [41] to be between 10 and 20\%. However, in certain scenarios ignoring the concrete's contribution is not satisfactory, so the shear coefficient for the whole section can be obtained from the following expression developed by Timoshenko [42]:

$$
\kappa^{-1}=\frac{A}{I^{2}} \int_{A} \frac{S^{2}(z)}{b(z)} d A
$$

where $A$ represents the total area of the beam, $I$ its corresponding principal moment of inertia, $S$ first moment of area at vertical coordinate $z$ (measured from the neutral axis), and $b(z)$ the measured width at the same coordinate $z$. Although several design guides recommend including all the time shear deformation effects in the analysis of flexural members, others specify that for span-to-depth ratios greater than 20 the shear influence can be neglected.

\subsubsection{Partial interaction}

If a partial interaction model is considered in the design, relative deformations are allowed at the interface between the PFRP profile and concrete slab. It is therefore necessary to know how the behavior of a hybrid beam is modified by the presence of slip. The shear-slip behavior between the FRP and concrete is problematic, presenting similar characteristics to the steel-concrete slip behavior. Nevertheless, studies [43,44] have shown that the shear-slip relation can be simplified as linear elastic under service load, in situations in which forces on connectors do not exceed about half their ultimate strength.

The current analytical model of a PFRP-RC hybrid beam with partial shear interaction is based on the following assumptions:

- shear connection stiffness is uniform and continuous along the interface;

- there is no vertical separation between the PFRP profile and the RC slab;

- Bernoulli’s hypotheses are applicable to both materials independently;

- $\quad$ the top steel reinforcement contribution is neglected;

- the whole width of the concrete slab is effective.

As before, the evaluation of deflections is performed under the elastic range of the beam's constitutive materials.

In the discussed analytical models the connected members are considered to be shear-rigid (the shear deformability is neglected for both connected layers) and therefore the partial interaction effects are quantified only from the perspective of the bending deflection $w_{b}$. 
The present study proposes the use of a dimensionless parameter $\xi$ that takes into account the influence of the reduced flexural stiffness due to the connection flexibility, by affecting the deflection due to bending under complete interaction assumptions:

$$
w_{b}^{p a}(x)=(1+\xi) \cdot w_{b}^{c o}(x)
$$

where the corresponding effective flexural rigidity is:

$$
E I_{e f f}=\frac{E I_{c o}}{1+\xi}
$$

The total deflection in the partial interaction model is obtained from:

$$
w_{t}^{p a}(x)=w_{b}^{p a}(x)+w_{s h}(x)
$$

By using an inverse approach it is possible to obtain $\xi$ from the deflection formulation suggested in an earlier version of Eurocode 4, for steel-concrete composite beams:

$$
\xi_{E C 4}=c(1-\eta)\left(\frac{E I_{c o}}{E_{p} I_{p}}-1\right)
$$

where $c$ is 0.5 for propped and 0.3 for unpropped construction, and $\eta$ represents the degree of partial shear connection based on the capacity of the connectors, as defined by the code. Similarly, a second expression for $\xi$ can be obtained from the equivalent flexural rigidity of composite beams discussed in Commentary part I3 of the American Specifications for Structural Steel Buildings ANSI/AISC 360-10:

$$
\xi_{A I S C}=\frac{E I_{c o}}{E_{p} I_{p}}\left[1+\sqrt{\eta}\left(\frac{E I_{c o}}{E_{p} I_{p}}-1\right)\right]^{-1}-1
$$

According to Eqs. (10) and (11), if a sufficient number of connectors is installed in a composite beam, i.e. $\eta=1$, then $\xi=$ 0 and the deflection is the same as under complete interaction assumptions. Nevertheless, research [45] has shown that even in these cases larger deflections than predicted may occur, and hence it is necessary to include the connection's flexibility in evaluating the partial interaction parameter $\xi$.

From push-out tests performed on shear connectors, a typical load ratio-slip response is registered. The slope of the initial linear elastic response is known as the connector stiffness $K_{c}$. Deriving a reliable design formulation for calculating $K_{c}$ presents difficulties and where experimental data is unavailable empirical formulations obtained from previous research may be used. In the case of steel-concrete composite beams with steel studs, after performing a large number of push-out test, Oehlers and Couglan [46] deducted the following expression from statistical interpretation:

$$
K_{c}=\frac{P_{\max }}{d\left(0.16-0.0017 f_{c}\right)}
$$

where $P_{\max }$ is the maximum capacity of a connector, $d$ its diameter, and $f_{c}$ represents the concrete slab's compressive strength. For timber composite beams, Eurocode 5 [47] suggests an empirical formulation for the connector's stiffness based on the density of the connected material, however during the validation procedure this second expression was found to be inappropriate for hybrid PFRP-RC beams so Eq. (12) is used subsequently throughout this study. 
Starting from a differential element of a PFRP-RC hybrid beam with partial interaction as depicted in Fig. 4, and by writing the appropriate equilibrium and compatibility equations based on the hypotheses mentioned before, one could arrive at the following two differential equations needed for determining the exact bending deflection $w_{b}^{p a}(x)$ :

$$
\begin{gathered}
\frac{\partial^{6}}{\partial x^{6}} w_{b}^{p a}(x)-\alpha^{2} \frac{\partial^{4}}{\partial x^{4}} w_{b}^{p a}(x)=\frac{1}{E I_{0}} \frac{\partial^{2}}{\partial x^{2}} q(x)-\alpha^{2} \frac{1}{E I_{c o}} q(x) \\
\frac{\partial^{2}}{\partial x^{2}} N(x)-\alpha^{2} N(x)=-\frac{K_{c} d_{c}}{S_{c} E I_{0}} M(x)
\end{gathered}
$$

where $M(x)$ is the total bending moment acting on the element, $\mathrm{N}(\mathrm{x})$ the axial force, $s_{c}$ the longitudinal spacing of the connectors, and $\alpha$ is given by:

$$
\alpha=\sqrt{\frac{K_{c}}{s_{c}} \frac{E I_{c o}}{E I_{0} \overline{E A}}}
$$

Exact solutions to differential Eqs. (13) and (14) can be found by applying suitable boundary conditions where, at specific coordinates along the beam, the deflection, slope or curvature have a known value. As a result, by employing a computational software program, the exact bending deflection formulations for the three static cases illustrated in Fig. 3 were determined and are summarized in Appendix A.

Using Eq. (7) and the relations in Appendix A, Table 1 summarizes the corresponding exact analytical expressions of the dimensionless partial interaction parameter $\xi$ and its maximum values in function of relative coordinates $x_{r}=x / L$.

Due to the complexity of the expressions for bending deflection considering partial interaction, various authors have suggested to use approximate solutions to the problem. By applying the proposed method from the current study, their formulations for effective flexural stiffness or effective maximum deflection were converted into the dimensionless parameter $\xi$.

In a study of steel-concrete composite beams considering shear slip effects [19], the authors suggest after a number of approximations the following formulation adapted here which was determined from the uniformly loaded beam case:

$$
\xi_{A}=\frac{12}{(\alpha L)^{2}}(\varphi+1)\left(0.4-\frac{3}{(\alpha L)^{2}}\right) ; \alpha L \geq 4
$$

A different research study presented in [48] suggests using in all cases the exact solution of the maximum deflection of a uniformly loaded simply supported steel-concrete composite beam with interlayer slip, irrespective of the boundary conditions. The following corresponding $\xi$ expression was determined from it:

$$
\xi_{B}=\frac{76.8}{(\alpha L)^{4}} \varphi\left[\operatorname{sech}\left(\frac{\alpha L}{2}\right)+\frac{(\alpha L)^{2}}{8}-1\right]=\xi_{3, \max }
$$

Similar investigations have been carried out on composite beams made of mechanically jointed timber layers or timber members connected to reinforced concrete with incomplete shear interaction. Annex B of Eurocode 5 offers an analytical model for calculating the effective flexural stiffness for this type of members, where the equation provided represents the exact solution for a simply supported beam with an applied sinusoidal load distribution. From its expression, the corresponding $\xi$ value was deducted: 


$$
\xi_{C}=\varphi\left[1+\left(\frac{\alpha L}{\pi}\right)^{2}\right]^{-1}
$$

Several studies [14,15] have adjusted the formulation for the effective flexural stiffness from Eurocode 5 by taking into account the effective length of the analyzed composite member as in the Euler buckling model. The same studies proved that differences between the effective $-\mu_{e f f}$ - and complete interaction $-\mu_{c o}$ - Euler buckling coefficients are minimal with a small exception for the pinned-clamped static case. Using Eq. (8), the dimensionless parameter obtained is:

$$
\xi_{D}=\frac{\mu_{e f f}^{2}}{\mu_{c o}^{2}} \varphi\left[1+\left(\frac{\mu_{e f f}}{\pi}\right)^{2}(\alpha L)^{2}\right]^{-1}
$$

For a simply supported composite beam $\mu_{e f f}=\mu_{c o}=1.00$ and the formulation reverts to Eq. (18).

One of the advantages of using this type of formulations as opposed to the exact ones is, besides their simplicity, the fact that the results are not sensitive to the load type and supporting conditions and thus the expressions can be regarded as generally valid.

\subsubsection{Parametric study}

In the following comparison charts, key parameters of the expressions presented before were varied in order to identify a single suitable approximate formulation for $\xi$ to be used in current design, which produces values sufficiently close to the exact formulations presented in Table 1.

The dimensionless parameter $\xi$ which quantifies the effects of the connection's flexibility in a hybrid beam's equation of deflection is mostly dependent on three dimensionless factors: the relative coordinate along the beam $x_{r}=x / L$, the composite action parameter $\alpha L$ and the relative bending stiffness parameter represented by the ratio $E I_{c o} / E I_{0}$. Because $\xi$ displays a linear variation in function of the ratio, a constant value of 2.5 was set for $E I_{c o} / E I_{0}$ for the whole parametric study, as averaged from the Table 4 ratios of investigated PFRP-RC hybrid beams with mechanical connections.

The variation of the exact and approximate formulations of $\xi$ for the three static cases illustrated in Fig. 3, in function of the relative longitudinal coordinates, is plotted in Fig. 5. In addition, the variation is considered for three distinct values of $\alpha L$ which imply that the connection has a lower or greater shear interaction degree. It is noted that as the connection's stiffness increases, the greater the $\alpha \mathrm{L}$, the plotted curves of the equations merge. Even for low interaction cases, errors in the increased bending deflection versus the exact formulations are smaller than $4 \%$ and for the maximum deflection are even less, with the exception of $\xi_{A}$.

A second comparison is made in Fig. 6 concerning the influence of the composite action parameter $\alpha L$ over $\xi$. The range of $\alpha L$ values is chosen to cover most of the practical scenarios and midspan values for $\xi$ are presented for the static cases depicted in Fig. 3. The discussed variation is also plotted against $\eta$, if the connection's flexibility is accounted for in the Eurocode 4 design model. The relation presented in the chart is highly nonlinear, whereas a linear increase in the composite action parameter significantly reduces the value of $\xi$. As indicated also by Fig. 5, Eq. (16) produces higher discrepancies compared to the others, especially for low interaction situations. 
Finally, opting for the simplicity and accuracy of Eq. (19), which in this case is identical to Eq. (18), the normalized effective bending deflection contribution is computed and plotted in Fig. 7 together with the normalized exact bending deflection formulations for the previously mentioned cases, considering complete and partial shear interaction behavior, against relative longitudinal coordinates. Normalization is performed by dividing the local expression with the maximum deflection under complete interaction conditions. Composite action parameter $\alpha L$ was fixed to 5 so as to highlight the fact that a weak connection between the PFRP profile and concrete slab may produce an almost 50\% increase in deflection.

In the end, the total deflection of a hybrid beam is much more sensible to the connection's stiffness $K_{c}$ and mechanical properties of the connected materials than to the approximations considered in evaluating the partial interaction parameter $\xi$, as it will be proved in the validation analysis.

\subsection{Interlayer slip}

Besides deflections, stress distributions in PFRP-RC beams are important to be determined especially for failure analysis. For a hybrid composite member with incomplete shear interaction, the first step in this analysis is to evaluate the slip and consequently the slip strain which develops at the interface of the connected materials, as seen in Fig. 2.

Once again, by writing the appropriate equilibrium and compatibility equations based on the hybrid differential element illustrated in Fig. 4 and on the hypotheses introduced in Section 2.1.2, the quadratic differential equation for the interlayer slip $s(x)$ can be written as:

$$
\frac{\partial^{2}}{\partial x^{2}} s(x)-\alpha^{2} s(x)=-\beta \alpha^{2} V(x)
$$

where $V(x)$ represents the shear force acting on the element and

$$
\beta=\frac{d_{c}}{\alpha^{2} E I_{0}}
$$

For the three static cases illustrated in Fig. 3, the general expressions for slip and slip strain were obtained by considering the relevant boundary conditions, and are presented in Appendix B. The slip strain $\varepsilon_{s}(x)$ equations were computed by differentiating the corresponding slip equations. Since the maximum values for slip and slip strain are important in estimating the flexural capacity of hybrid beams with partial interaction, Table 2 summarizes their expressions.

The main parameters on which slip and slip strain rely are the position along the beam, parameter $\beta$, the dimensionless composite action parameter $\alpha L$, and the applied load (in a direct linear variation). The normalized longitudinal distributions of slip and slip strain for the analyzed static cases are plotted in Fig. 8. The distribution profiles are highly nonlinear, with maximum slip values occurring at the supports and maximum slip strains showing up at critical, maximum bending moment sections. 


\subsection{Flexural capacity}

Using the constitutive models of the hybrid beam's materials, as described in the introductory part of Section 2 and depicted in Fig. 2, coupled with the assumptions enumerated in Sections 2.1.1 and 2.1.2, the analytical expressions for the flexural capacity of hybrid beams with full and partial shear connection were evaluated. The following corresponding sections refer to the most realistic case in practice, where the neutral axis crosses the top concrete slab, considering that a composite profile acting both in compression and tension would be undesired from a stability point of view.

\subsubsection{Complete interaction}

If the connection is capable of transmitting entirely the axial force developed in the reinforced concrete slab to the PFRP profile and if the neutral axis lays inside the concrete layer as seen in Fig. 2(a), the depth of the neutral axis $x_{u}$ found from the equilibrium of the cross-section is expressed by:

$$
0.8 b_{c} \frac{f_{c}}{\varepsilon_{c, u}} x_{u}^{2}+A_{p} E_{p} x_{u}-A_{p} E_{p}\left(\frac{h_{p}}{2}+h_{c}\right)=0
$$

Therefore, the maximum bending moment that the hybrid beam can sustain, considering crushing of the concrete slab and a full shear connection, is computed from:

$$
M_{u, c o}=0.6 x_{u} F_{c}+F_{f t}\left(h_{c}-x_{u}+\frac{t_{f}}{2}\right)+F_{w}\left(h_{c}-x_{u}+\frac{h_{p}}{2}\right)+F_{f b}\left(h-x_{u}-\frac{t_{f}}{2}\right)
$$

where the forces acting in the concrete slab $F_{c}$, profile's top flange $F_{f t}$, web $F_{w}$, and bottom flange $F_{b f}$ are:

$$
\begin{gathered}
F_{c}=0.8 x_{u} b_{c} f_{c} \\
F_{f t}=A_{f} E_{p} \frac{\varepsilon_{c, u}}{x_{u}}\left(h_{c}-x_{u}+\frac{t_{f}}{2}\right) \\
F_{w}=A_{w} E_{p} \frac{\varepsilon_{c, u}}{x_{u}}\left(h_{c}-x_{u}+\frac{h_{p}}{2}\right) \\
F_{f b}=A_{f} E_{p} \frac{\varepsilon_{c, u}}{x_{u}}\left(h-x_{u}-\frac{t_{f}}{2}\right)
\end{gathered}
$$

where $A_{f}$ represents the profile's flange area and $A_{w}$ its web area. The rest of the geometric parameters are described in Fig. 2.

\subsubsection{Partial interaction}

For hybrid beams with partial shear connection, where a relative slip develops at the interface and the neutral axis is lying in the concrete layer as shown in Fig. 2(b), the depth of the neutral axis depends in addition on the slip strain $\varepsilon_{s}$, and can be determined from the following equilibrium equation adapted from [32]:

$$
0.8 b_{c} f_{c} x_{u}^{2}+A_{p} E_{p}\left(\varepsilon_{c, u}+\varepsilon_{s}\right) x_{u}-A_{p} E_{p} \varepsilon_{c, u}\left(\frac{h_{p}}{2}+h_{c}\right)=0
$$


Hence, the maximum bending moment that the hybrid beam can sustain, considering crushing of the concrete slab and incomplete connection, is:

$$
M_{u, p a}=0.6 x_{u} F_{c}^{p a}+F_{f t}^{p a}\left(h_{c}-x_{u}+\frac{t_{f}}{2}\right)+F_{w}^{p a}\left(h_{c}-x_{u}+\frac{h_{p}}{2}\right)+F_{f b}^{p a}\left(h-x_{u}-\frac{t_{f}}{2}\right)
$$

where the forces acting in the concrete slab $F_{c}^{p a}$, profile’s top flange $F_{f t}^{p a}$, web $F_{w}^{p a}$, and bottom flange $F_{b f}^{p a}$ are:

$$
\begin{gathered}
F_{c}^{p a}=0.8 x_{u} b_{c} f_{c} \\
F_{f t}^{p a}=F_{f t}-A_{f} E_{p} \varepsilon_{s} \\
F_{w}^{p a}=F_{w}-A_{w} E_{p} \varepsilon_{s} \\
F_{f b}^{p a}=F_{f b}-A_{f} E_{p} \varepsilon_{s}
\end{gathered}
$$

Using a different approach [19], by considering that the flexural moment is the sum between the flexural moment for the same hybrid beam but with complete connection and the negative moment produced by the couple between the axial forces developed from partial interaction, the decrease in flexural capacity due to the flexibility of the connection system (i.e. slip) is:

$$
\Delta M(x)=\frac{h_{p}}{6 h} E_{p}\left(2 h_{c} A_{f}+h A_{w}\right) \varepsilon_{s}(x)
$$

Because the slip strain equation has to be solved afresh for each force increment and different boundary conditions, the formulation is cumbersome for routine design. In exchange, by expressing the curvature increase of the beam through the proposed dimensionless partial interaction parameter $\xi$ as below:

$$
\Delta \phi(x)=\frac{\varepsilon_{s}(x)}{h}=\frac{M_{c o}(x)}{E I_{c o}} \xi(x)
$$

a simple, yet reliable formula may be obtained for the effective flexural capacity of hybrid beams with partial shear connection:

$$
M_{u, e f f}=M_{u, c o}-\Delta M=M_{u, c o}\left[1-\xi_{\max } \frac{h_{p} E_{p}}{6 E I_{c o}}\left(2 h_{c} A_{f}+h A_{w}\right)\right]
$$

\subsection{Axial and shear stresses}

\subsubsection{Complete interaction}

For hybrid beams with full composite action, the normal stress in a section can be computed using Navier's formula:

$$
\sigma_{j, c o}(x, z)=\mp \frac{M(x) E_{j}}{E I_{c o}} z
$$

where subscript $j$ defines the material layer ( $c$ for concrete and $p$ for the PFRP profile), $x$ the longitudinal coordinate and $Z$ the distance in the $Z$ direction from the section's neutral axis to the point of interest. The minus sign corresponds to the top layer while the plus sign refers to the bottom layer. Maximum axial deformations are found at the extremities and even 
though the distribution is considered to be linear, warping of the PFRP profile may occur especially in short, stocky members.

The shear stress in a PFRP-RC beam can be obtained from the Jourawski-Collignon formula:

$$
\tau_{j, c o}(x, z)=\frac{V(x) E_{j} S(z)}{E I_{c o} b_{j}(z)}
$$

where the shear force $V(x)$ is considered positive, $S(z)$ represents the first moment of area calculated at coordinate $z$, and $b_{j}(z)$ is the width of material layer $j$ measured at $z$. Maximum shear stresses usually occur at the position of the hybrid beam's neutral axis or towards the interface between the connected elements.

\subsubsection{Partial interaction}

In the case of hybrid beams with partial shear connection, where the connection's flexibility affects the stress distributions in the structural member, Eqs. (37) and (38) can be adapted in an approximate analysis [15] by substituting the flexural rigidity under complete connection assumptions with an effective flexural stiffness determined from Eq. (8).

Thus, the expression for normal stress becomes:

$$
\sigma_{j, e f f}\left(x, z_{j}\right)=\left[\mp\left(1-\frac{1+\xi}{1+\varphi}\right) \frac{1}{A_{j} d_{c}}+(1+\xi) \frac{E_{j} z_{j}}{E I_{c o}}\right] M(x)
$$

where $z_{j}$ represents the coordinate measured from the centroid of layer $j$ towards the calculation point (in the $Z$ direction), and $A_{j}$ is the transversal area of sub-element $j$. The minus and plus signs are again applied as specified for Eq. (37).

The shear stress distribution is calculated from:

$$
\tau_{j, e f f}\left(x, z_{j}\right)=\left[\left(1-\frac{1+\xi}{1+\varphi}\right) \frac{A_{s h, j}\left(z_{j}\right)}{A_{j} d_{c} b\left(z_{j}\right)}+(1+\xi) \frac{E_{j} S\left(z_{j}\right)}{E I_{c o} b\left(z_{j}\right)}\right] V(x)
$$

where the sheared area of layer $j$, denoted $A_{s h, j}\left(z_{j}\right)$, the first moment of area $S\left(z_{j}\right)$ and the width $b\left(z_{j}\right)$ are all determined using local coordinates $z_{j}$.

\subsubsection{Stress failure checks}

For design purposes, the stresses developing in a PFRP-RC hybrid beam at the Ultimate Limit State (ULS) have to be checked against allowable values such as material strengths. Because of the nature of composite materials, maximum longitudinal stresses occurring in the flanges of the profile are usually way lower than the corresponding strength, while maximum normal transverse stress and in-plane shear stress values are regarded as critical.

Due to the anisotropy of the composite material, pultruded profiles are susceptible to crushing failure at the reaction points and under concentrated loads. Its compressive strength in transverse direction $\sigma_{c M, T}$ is about five times lower than in the longitudinal direction. Thus, the critical crushing force can be calculated using the following expression: 


$$
F_{\text {crush }}=\sigma_{c M, T} A_{e f f}
$$

where $A_{\text {eff }}$ is the effective area over which the force is applied.

Assuming the conservative hypothesis that shear stress in a hybrid beam is entirely carried by the profile's web, and its distribution is relatively uniform along the depth of the web, the critical shear force for a homogeneous profile due to web material failure is:

$$
V_{\text {max }} \approx \tau_{\text {max }} A_{\text {sh }}
$$

where $\tau_{\max }$ is the in-plane shear strength of the PFRP profile and $A_{s h}$ is the sheared area approximated as:

$$
A_{s h} \approx t_{w}\left(d_{w}+t_{f}\right)
$$

Often times the in-plane shear strength of GFRP profiles is not reported by manufacturers due to the complexity involved by the experimental determination of this property. Nevertheless, the interlaminar (or out-of-plane) shear strength is usually reported and can be used instead. Being about 30\% smaller than the in-plane shear strength, the calculation will lead to overly conservative designs for conventional pultruded profiles.

\section{Validation of analytical models}

\subsection{Description of specimens}

To assert the validity of the analytical models presented before, the published characteristics and experimental results of six PFRP-RC hybrid beams were used in the current investigation. The chosen specimens featured only mechanical connections with either steel bolts or GFRP dowels, had different spans, load conditions and cross-section geometries (see Fig. 2 for a description of parameters). In addition to dimension ratios, Table 3 summarizes experimental values of the connection stiffness $K_{c}^{\text {exp }}$ where push-out tests were performed, and corresponding analytical values determined from Eq. (12). The percentile difference $\Delta$ between the latter two columns proves to be conservative.

The dimension ratios reveal that both slender and stocky beam types were taken into consideration for the analysis, with specimen HB3 having the smallest shear span-to-depth ratio. According to Eurocode 4, the high degree of shear connection $\eta$ suggests that the beams had full shear interaction, however during the reported tests larger deflections and slippage at the interface were noticed in all cases. Therefore, the continuing analysis under partial interaction is based on the connection's flexibility reflected by the modulus $K_{c}$.

With the help of the relations provided in Section 2, the main parameters of partial interaction were computed for each hybrid specimen and presented in Table 4. The beams have a low to medium degree of composite action so the reduction in the flexural rigidity varies between $13 \%$ and $39 \%$. At the same time, the error $\Delta$ between the exact value of $\xi_{i, \max }$ depending on the static case $i$, as determined from the equations in Table 1 and the approximate value $\xi_{D}$ obtained from Eq. (19) appears to be negligible.

Participation ratios of the internal moments and forces acting in the hybrid beam sub-systems were computed in Table 5 considering a linear elastic behavior of the materials, for both complete and incomplete connection cases. The numbers 
indicate a significant reduction up to $26 \%$ in the coupling moment $N d_{c}$ between the two layers, for the partial connection model. As a result the individual moments carried by the profile $M_{p}$ and concrete slab $M_{c}$ increase significantly. The percentile differences regarding the shear force in the two layers are by comparison not so important, however if the reinforced concrete element carries a large part of it, then instead of using the approximate Eqs. (5) and (42) in evaluating the deflection and shear stress, Eqs. (6), and (38) or (40) are recommended.

\subsection{Serviceability analysis}

Based on a survey [49] of the main guides and manuals for the design of structures using PFRP elements, a maximum value for the deflection under serviceability conditions has been suggested equal to $L / 250$ for quasi-permanent load combination for floors, that is used in the current validation routine.

Considering models with both complete and partial shear interaction under different hypotheses and given the fixed experimental deflection $w_{t}^{\text {exp }}$, the maximum total load $Q_{t}$ acting on each beam is computed and presented in Table 6 together with the percentile difference versus the experimental value.

The numbers suggest a minimal error between the exact and effective partial model, and an acceptable one versus the experimental data. However, the difference increases when simplifying the model by considering that only the web of the profile carries shear.

\subsection{Failure analysis}

As observed from Table 7, the main reported failure mode of the investigated hybrid beams was characterized by the loss of shear strength capacity in the web of the PFRP profile. Nevertheless, maximum bending capacities were calculated for each specimen according to Eqs. (29) and (36), assuming that the concrete slabs crushes under compression, and results show a tolerable difference $\Delta$ between using the complex formulations including slip and the more robust, approximate formulation of using the dimensionless parameter of partial interaction $\xi$. By using Eqs. (40) and (42) for determining the critical in-plane shear stress, the calculated maximum flexural moments limited by the shear load, $M_{u, p a}^{s h}$, are represented in Table 7 as percentages of the capacities considering the previously mentioned concrete crushing hypothesis.

Once more, after studying models with both complete and partial shear connection under different hypotheses and provided the experimental results, the analytical values for the total acting loads $Q_{t}$ and midspan deflections $w_{t}$ are grouped in Table 8 together with the difference in percentage versus the experimental values.

Results show that partial interaction formulations model better the flexural response of the hybrid beams compared to the complete interaction equations and deflection values are underestimated due to the fact that concrete has a profound nonlinear response closer to its maximum strength. The analysis also reveals that deflections obtained considering only the shear deformation contributions of the profile’s web are more conservative, as expected. 


\subsection{Capacity-deflection comparison}

Fig. 9 plots the experimental and analytical load-midspan deflection curves obtained for the hybrid beam specimens, considering incomplete and full composite action. For the partial interaction model, approximate values of the dimensionless parameter $\xi$ were used, as specified in Table 4. Results indicate that by including the slip effects in the design of PFRP-RC beams, better predictions can be made as the displayed effective flexural stiffness is closer to the experimentally recoded response. Nevertheless, for specimens No1 and No2 the complete interaction curves are more accurate probably because of the empirical nature of Eq. (12) used to determine the flexibility of the connection system.

The validation diagram presented in Fig. 10 confirms that the ultimate bending capacity $M_{u}$ as well as the corresponding ultimate midspan deflection $w_{u}$, using the formulations for the simplified model with partial connection, are more accurate than the analytical model with complete shear connection.

\subsection{Strain and stress distributions}

A final comparison is made by validating the available experimental strain and stress distributions of the hybrid beams with the results computed from the equations discussed in Section 2.4.2. Firstly, the load-strain curves obtained from cross-sectional strain gauge measurements on the profiles and concrete slabs are compared against analytical values calculated with Eq. (39) under partial interaction premises. The plots depicted in Fig. 11 prove that the analytical model is sufficiently accurate.

Secondly, Eqs. (38) and (40) which calculate the complete and effective in-plane shear stresses were used to plot the sectional stress distributions for hybrid beams specimen HB1 in Fig. 12. The diagrams were validated against the experimental data measured at the instrumented location $\tau_{m, \max }$ and the strength of the pultruded composite material $\tau_{\max }$.

As indicated in Table 6, HB1 ultimately failed due to shearing of the profile's web, a fact also confirmed accurately by the analytical maximum effective in-plane shear stress distribution. In addition, Fig. 12 supports the hypothesis that the majority of the shear force is carried by the hybrid beam's profile web, as approximated in Eqs. (5) and (43).

\section{Conclusions}

Studies performed on hybrid beams so far have rarely included the influence of the connection's stiffness over flexural behavior. The current paper presented a detailed analytical design procedure suitable for hybrid beams made of pultruded FRP profiles connected to reinforced concrete slabs considering both complete and partial shear interaction scenarios. The following main observations are made:

i. A dimensionless parameter is introduced to account for the shear interaction effects, which depends mainly on the connectors' stiffness and the flexural rigidity characteristics of the hybrid member. 
ii. After performing a parametric study, the simplified expression of the introduced parameter, derived from the Eurocode 5 method, is found to be the most suitable for current design due to its accuracy and independence to the load and supporting conditions.

iii. Exact and simplified expressions are detailed for calculating deflections, stiffness, slip and bending capacities, as well as normal and shear stress distributions for hybrid PFRP-RC beams.

iv. Experimental data from four separate investigations are successfully matched with analytical results compiled for load-displacement curves, axial strain variations and shear stress profiles.

v. The validation analysis highlights one of the issues that hybrid beams possess, that the flexural capacity is usually limited by the shear strength of the composite material, thus the structural element should be designed to have a pseudo-ductile compressive concrete failure before.

vi. Deflection and shear stress results justify the conservative approach in assuming that the entire shear force is carried by the profile’s web.

The analytical procedure should be used for the design of hybrid PFRP-RC beams regardless of the amount of shear connection provided because partial interaction effects can lead to substantially higher deflections, reduced flexural capacities and lower shear stress values.

As a future task the authors would like to validate the proposed analytical procedure against experimental results of hybrid beams with a low degree of shear connection. Furthermore, a reliable method for obtaining the stiffness modulus of connectors for hybrid beams should be developed as this property greatly influences the results considering partial interaction.

\section{Acknowledgements}

The presented work is part of the research project COMPOBEAM - Researching the flexural behavior of mixed beams made of concrete and GFRP profiles, developed at CER LITEM/UPC-BarcelonaTech. The authors would like to acknowledge the financial support from company PIGRA Engineering S.L. through CDTI. The first author is also grateful for the financial aid provided by the FPI-UPC doctoral scholarship.

\section{Appendix A. Exact bending deflection formulations for specific cases}

The exact closed-form solutions to the bending deflection contributions of hybrid beams with partial shear interaction were obtained using a computational software program, Mathematica, for the three static cases presented in Fig. 3, by applying appropriate boundary conditions to differential Eqs. (13) and (14).

For Fig. 3(a), the exact bending deflection and maximum bending deflection solutions are as follows: 


$$
\begin{gathered}
w_{b, 1}^{p a}(x)=w_{b, 1}^{c o}(x)+\frac{Q}{2 \alpha^{3} E I_{c o}} \varphi\left[\alpha x-\operatorname{sech}\left(\frac{\alpha L}{2}\right) \sinh (\alpha x)\right] ; x \in[0, L / 2] \\
w_{b, 1, \max }^{p a}=\frac{Q L^{3}}{48 E I_{c o}}+\frac{Q}{2 \alpha^{3} E I_{c o}} \varphi\left[\frac{\alpha L}{2}-\tanh \left(\frac{\alpha L}{2}\right)\right]
\end{gathered}
$$

for Fig. 3(b):

$$
\begin{gathered}
w_{b, 2}^{p a}(x)=w_{b, 2}^{c o}(x)+\frac{Q}{\alpha^{3} E I_{c o}} \varphi\left\{\alpha x+\sinh (\alpha x)\left[\sinh (\alpha b) \tanh \left(\frac{\alpha L}{2}\right)-\cosh (\alpha b)\right]\right\} ; x \in[0, b] \\
w_{b, 2}^{p a}(x)=w_{b, 2}^{c o}(x)+\frac{Q}{\alpha^{3} E I_{c o}} \varphi\left\{\alpha b+\sinh (\alpha b)\left[\sinh (\alpha x) \tanh \left(\frac{\alpha L}{2}\right)-\cosh (\alpha x)\right]\right\} ; x \in[b, L / 2] \\
w_{b, 2, \max }^{p a}=\frac{Q b}{24 E I_{c o}}\left(3 L^{2}-4 b^{2}\right)+\frac{Q}{\alpha^{3} E I_{c o}} \varphi\left[\alpha b-\sinh (\alpha b) \operatorname{sech}\left(\frac{\alpha L}{2}\right)\right]
\end{gathered}
$$

and for Fig. 3(c):

$$
\begin{gathered}
w_{b, 3}^{p a}(x)=w_{b, 3}^{c o}(x)+\frac{q_{0}}{\alpha^{4} E I_{c o}} \varphi\left\{\cosh \left[\alpha\left(x-\frac{L}{2}\right)\right] \operatorname{sech}\left(\frac{\alpha L}{2}\right)-\frac{(\alpha x)^{2}}{2}+\frac{\alpha^{2} L x}{2}-1\right\} ; x \in[0, L / 2] \\
w_{b, 3, \max }^{p a}=\frac{5 q_{0} L^{4}}{384 E I_{c o}}+\frac{q_{0}}{\alpha^{4} E I_{c o}} \varphi\left[\operatorname{sech}\left(\frac{\alpha L}{2}\right)+\frac{(\alpha L)^{2}}{8}-1\right]
\end{gathered}
$$

\section{Appendix B. Slip and slip strain expressions for specific cases}

The slip solutions for the second order differential Eq. (20) were determined for the three static cases illustrated in Fig. 3 by considering that no slip occurs at the midspan and slip strain is zero at the ends of the hybrid beams. Subsequently, slip strain equations were differentiated from the slip expressions and are presented below.

For the static case illustrated in Fig. 3(a), where $x \in[0, L / 2]$, the expressions are:

$$
\begin{gathered}
s_{1}(x)=\beta \frac{Q}{2}\left[1-\frac{\cosh (\alpha x)}{\cosh (\alpha b)}\right] \\
\varepsilon_{s, 1}(x)=\alpha \beta \frac{Q}{2} \frac{\sinh (\alpha x)}{\cosh (\alpha b)}
\end{gathered}
$$

for Fig 3(b), when $x \in[0, b]$ :

$$
\begin{gathered}
s_{2}(x)=\beta Q\left\{1-\operatorname{sech}\left(\frac{\alpha L}{2}\right) \cosh \left[\alpha\left(\frac{L}{2}-b\right)\right] \cosh (\alpha x)\right\} \\
\varepsilon_{s, 2}(x)=\alpha \beta Q\left\{\operatorname{sech}\left(\frac{\alpha L}{2}\right) \cosh \left[\alpha\left(\frac{L}{2}-b\right)\right] \sinh (\alpha x)\right\}
\end{gathered}
$$

and when $x \in[b, L / 2]$

$$
\begin{gathered}
s_{2}(x)=\beta Q\left\{\operatorname{sech}\left(\frac{\alpha L}{2}\right) \sinh \left[\alpha\left(\frac{L}{2}-x\right)\right] \sinh (\alpha b)\right\} \\
\varepsilon_{s, 2}(x)=\alpha \beta Q\left\{\operatorname{sech}\left(\frac{\alpha L}{2}\right) \cosh \left[\alpha\left(\frac{L}{2}-x\right)\right] \sinh (\alpha b)\right\}
\end{gathered}
$$

respectively for Fig 3(c), where $x \in[0, L / 2]$ : 


$$
\begin{gathered}
s_{3}(x)=\beta q_{0}\left\{\left(\frac{L}{2}-x\right)-\frac{1}{\alpha} \operatorname{sech}\left(\frac{\alpha L}{2}\right) \sinh \left[\alpha\left(\frac{L}{2}-x\right)\right]\right\} \\
\varepsilon_{s, 3}(x)=\beta q_{0}\left\{1-\operatorname{sech}\left(\frac{\alpha L}{2}\right) \cosh \left[\alpha\left(\frac{L}{2}-x\right)\right]\right\}
\end{gathered}
$$

\section{References}

[1] Kollár LP. Local Buckling of Fiber Reinforced Plastic Composite Structural Members with Open and Closed Cross Sections. J Struct Eng 2003;129:1503-13. doi:10.1061/(ASCE)0733-9445(2003)129:11(1503).

[2] Ascione L, Feo L, Mancusi G. On the statical behaviour of fibre-reinforced polymer thin-walled beams. Compos Part B Eng 2000;31:643-54. doi:10.1016/S1359-8368(00)00032-9.

[3] Deskovic N, Triantafillou TC, Meier U. Innovative Design of FRP Combined with Concrete: Short-Term Behavior. J Struct Eng 1995;121:1069-78. doi:10.1061/(ASCE)0733-9445(1995)121:7(1069).

[4] Canning L, Hollaway L, Thorne AM. An investigation of the composite action of an FRP/concrete prismatic beam. Constr Build Mater 1999;13:417-26. doi:10.1016/S0950-0618(99)00050-1.

[5] Hall JE, Mottram JT. Combined FRP Reinforcement and Permanent Formwork for Concrete Members. J Compos Constr 1998;2:78-86. doi:10.1061/(ASCE)1090-0268(1998)2:2(78).

[6] Mirmiran A. Innovative combinations of FRP and traditional materials. In: Teng JG, editor. Int. Conf. FRP Compos. Civ. Eng., vol. II, Hong Kong, China: Elsevier Science Ltd.; 2001, p. 1289-98.

[7] Mendes Ferreira AJ, Ribeiro MCS, Torres Marques A. Analysis of hybrid beams composed of GFRP profiles and polymer concrete. Int J Mech Mater Des 2004;1:143-55.

[8] Mutsuyoshi H, Hai ND, Wu Z. Structural Behavior of Composite Girders Consisting of Hybrid FRP I-Beam and Precast Ultra High Performance Fiber Reinforced Concrete Slab. Third Asia-Pacific Conf. FRP Struct., Hokkaido, Japan: 2012.

[9] Neagoe CA, Gil L. Experimental study of GFRP-concrete hybrid beams with partial interaction. B. Abstr. - 17th Int. Conf. Compos. Struct., Porto, Portugal: 2013, p. 153.

[10] Clarke JL, editor. Structural Design of Polymer Composites - EUROCOMP Design Code and Handbook. E \& FN SPON; 1996.

[11] Johnson RP. Composite Structures of Steel and Concrete. Third Ed. Oxford, UK: Blackwell Publishing Ltd; 2004. doi:10.1002/9780470774625.

[12] CEN. Eurocode 4: Design of composite steel and concrete structures. Part 1-1: General rules and rules for buildings. EN 1994-1-1:2004. Brussels, Belgium: 2004.

[13] AISC. Specification for Structural Steel Buildings. ANSI/AISC 360-10. Chicago, Illinois, USA: 2010.

[14] Girhammar UA, Gopu VKA. Composite Beam-Columns with Interlayer Slip-Exact Analysis. J Struct Eng 1993;119:1265-82. doi:10.1061/(ASCE)0733-9445(1993)119:4(1265).

[15] Girhammar UA. A simplified analysis method for composite beams with interlayer slip. Int J Mech Sci 2009;51:515-30. doi:10.1016/j.ijmecsci.2009.05.003. 
[16] Girhammar UA, Pan DH, Gustafsson A. Exact dynamic analysis of composite beams with partial interaction. Int J Mech Sci 2009;51:565-82. doi:10.1016/j.ijmecsci.2009.06.004.

[17] Faella C, Martinelli E, Nigro E. Steel and concrete composite beams with flexible shear connection: "exact" analytical expression of the stiffness matrix and applications. Comput Struct 2002;80:1001-9. doi:10.1016/S00457949(02)00038-X.

[18] Faella C, Martinelli E, Nigro E. Shear Connection Nonlinearity and Deflections of Steel-Concrete Composite Beams: A Simplified Method. J Struct Eng 2003;129:12-20. doi:10.1061/(ASCE)0733-9445(2003)129:1(12).

[19] Nie J, Cai CS. Steel-Concrete Composite Beams Considering Shear Slip Effects. J Struct Eng 2003;129:495-506. doi:10.1061/(ASCE)0733-9445(2003)129:4(495).

[20] Nie J, Fan J, Cai CS. Stiffness and Deflection of Steel-Concrete Composite Beams under Negative Bending. J Struct Eng 2004;130:1842-51. doi:10.1061/(ASCE)0733-9445(2004)130:11(1842).

[21] Nie J, Fan J, Cai CS. Experimental study of partially shear-connected composite beams with profiled sheeting. Eng Struct 2008;30:1-12. doi:10.1016/j.engstruct.2007.02.016.

[22] Frangi A, Fontana M. Elasto-Plastic Model for Timber-Concrete Composite Beams with Ductile Connection. Struct Eng Int 2003;13:47-57. doi:10.2749/101686603777964856.

[23] Persaud R, Symons D. Design and testing of a composite timber and concrete floor system. J Struct Eng Soc New Zeal 2006;19:30-43.

[24] Schnabl S, Saje M, Turk G, Planinc I. Analytical Solution of Two-Layer Beam Taking into account Interlayer Slip and Shear Deformation. J Struct Eng 2007;133:886-94. doi:10.1061/(ASCE)0733-9445(2007)133:6(886).

[25] Xu R, Wu Y. Static, dynamic, and buckling analysis of partial interaction composite members using Timoshenko’s beam theory. Int J Mech Sci 2007;49:1139-55. doi:10.1016/j.ijmecsci.2007.02.006.

[26] Xu R, Wang G. Bending Solutions of the Timoshenko Partial-Interaction Composite Beams Using Euler-Bernoulli Solutions. J Eng Mech 2013;139:1881-5. doi:10.1061/(ASCE)EM.1943-7889.0000614.

[27] Martinelli E, Nguyen QH, Hjiaj M. Dimensionless formulation and comparative study of analytical models for composite beams in partial interaction. J Constr Steel Res 2012;75:21-31. doi:10.1016/j.jcsr.2012.02.016.

[28] Sekijima K, Miyata K, Ihara T, Hayashi K. Study on Flexural Behavior of Fiber Reinforced Plastic-Concrete Composite Beam. Third Int. Symp. Non-Metallic Reinf. Concr. Struct., Sapporo, Japan: Japan Concrete Institute; 1997, p. 543-50.

[29] Sekijima K, Ogisako E, Miyata K, Hayashi K. Analytical study on flexural behavior of GFRP-concrete composite beam. In: Teng JG, editor. Int. Conf. FRP Compos. Civ. Eng., vol. II, Hong Kong, China: Elsevier Science Ltd.; 2001, p. 1363-70.

[30] Biddah A. Experimental investigation of pultruded FRP section combined with concrete slab. In: Tan KH, editor. Sixth Int. Symp. FRP Reinf. Concr. Struct., Singapore: World Scientific Publishing Co. Pte. Ltd.; 2003.

[31] Fam A, Skutezky T. Composite T-Beams Using Reduced-Scale Rectangular FRP Tubes and Concrete Slabs. J Compos Constr 2006;10:172-81. doi:10.1061/(ASCE)1090-0268(2006)10:2(172).

[32] Correia JR, Branco FA., Ferreira JG. Flexural behaviour of GFRP-concrete hybrid beams with interconnection slip. Compos Struct 2007;77:66-78. doi:10.1016/j.compstruct.2005.06.003. 
[33] Correia JR, Branco FA., Ferreira J. GFRP-concrete hybrid cross-sections for floors of buildings. Eng Struct 2009;31:1331-43. doi:10.1016/j.engstruct.2008.04.021.

[34] Knowles PR. Composite Steel and Concrete Construction. Wiley; 1973.

[35] CEN. Eurocode 2: Design of concrete structures. Part 1-1: General rules and rules for buildings. EN 1992-11:2004. Brussels, Belgium: 2004.

[36] Bank LC. Shear coefficients for thin-walled composite beams. Compos Struct 1987;8:47-61. doi:10.1016/02638223(87)90015-8.

[37] Barbero EJ, Lopez-Anido R, Davalos JF. On the Mechanics of Thin-Walled Laminated Composite Beams. J Compos Mater 1993;27:806-29. doi:10.1177/002199839302700804.

[38] Omidvar B. Shear Coefficient in Orthotropic Thin-Walled Composite Beams. J Compos Constr 1998;2:46-56. doi:10.1061/(ASCE)1090-0268(1998)2:1(46).

[39] Hutchinson JR. Shear Coefficients for Timoshenko Beam Theory. J Appl Mech 2001;68:87. doi:10.1115/1.1349417.

[40] Roberts TM, Al-Ubaidi H. Flexural and Torsional Properties of Pultruded Fiber Reinforced Plastic I-Profiles. J Compos Constr 2002;6:28-34. doi:10.1061/(ASCE)1090-0268(2002)6:1(28).

[41] Bank LC. Composites for construction: Structural design with FRP materials. Hoboken, New Jersey: John Wiley \& Sons, Inc.; 2006.

[42] Gere JM, Timoshenko SP. Mechanics of Materials. 3rd ed. Pws Pub Co; 1990.

[43] Stüssi F. Contribution to working meeting VII A 4. Final Rep. First Congr. Int. Assoc. Bridg. Struct. Eng., Paris: 1932, p. 579-95.

[44] Newmark NM, Siess CP, Viest IM. Test and analysis of composite beams with incomplete interaction. Proc Soc Exp Stress Anal 1951;9:75-92.

[45] Neagoe CA, Gil L. Evaluation of Deflections for PFRP-RC Hybrid Beams with Complete and Partial Shear Connection. In: Chiorean CG, editor. Proc. Second Int. Conf. PhD Students Civ. Eng. Archit. (CE-PhD 2014), Cluj-Napoca, Romania: UTPRESS; 2014, p. 57-64.

[46] Oehlers DJ, Coughlan CG. The shear stiffness of stud shear connections in composite beams. J Constr Steel Res 1986;6:273-84. doi:10.1016/0143-974X(86)90008-8.

[47] CEN. Eurocode 5: Design of timber structures. Part 1-1: General rules and rules for buildings. EN 1995-1-1:2004. Brussels, Belgium: 2004.

[48] Wang YC. Deflection of Steel-Concrete Composite Beams with Partial Shear Interaction. J Struct Eng 1998;124:1159-65. doi:10.1061/(ASCE)0733-9445(1998)124:10(1159).

[49] Neagoe CA. Design and analysis of PFRP and concrete hybrid beams. In: Pérez MA, editor. Adv. Appl. Compos. Mater. Civ. Work. Build., OmniaScience Monographs; 2014, p. 205-36. doi:10.3926/oms.207 [in Spanish].

\section{Figure captions}

Fig. 1. Flexural behavior of a hybrid beam with complete or partial shear interaction. 
Fig. 2. Generic hybrid cross-section geometry with corresponding strain $(\varepsilon)$ and stress $(\sigma)$ distributions considering: (a) complete or (b) partial shear interaction; at ULS.

Fig. 3. Static cases analyzed. Simply-supported hybrid beams subjected to: (a) a concentrated midspan load; (b) two symmetrically applied loads; (c) a uniform load.

Fig. 4. Differential element for a hybrid PFRP-RC beam with partial interaction.

Fig. 5. Variation of partial interaction parameter $\xi$ to relative coordinates.

Fig. 6. Influence of composite action parameter $\alpha L$ over $\xi$, at midspan.

Fig. 7. Variation of complete and partial normalized deflections to relative coordinates.

Fig. 8. Normalized longitudinal distributions of slip and slip strain for the static cases illustrated in Fig. 3.

Fig. 9. Experimental and analytical load-deflection curves of hybrid beam specimens. Partial and complete interaction considered.

Fig. 10. Validation diagram for the analytical model in terms of flexural capacity and maximum deflection.

Fig. 11. Experimental and analytical load-strain curves of hybrid beam specimens. Only partial interaction considered.

Fig. 12. Analytical in-plane shear stress distribution over the depth of hybrid beam HB1.

\section{Tables}

Table 1. Exact analytical expressions for partial interaction parameter $\xi$ and corresponding maximum values.

Table 2. Analytical solutions for the maximum slip and slip strain.

Table 3. Characteristics of the hybrid beam specimens chosen for validation analysis.

Table 4. Computed parameters of partial interaction.

Table 5. Participation percentages of hybrid beam sub-systems.

Table 6. Maximum loads considering various hypotheses at SLS.

Table 7. Experimental failure characteristics and maximum computed moments.

Table 8. Maximum loads and total deflections considering various hypotheses at ULS. 